\title{
The Utility of EEG, SSEP, and Other Neurophysiologic Tools to Guide Neurocritical Care
}

\author{
Eric S. Rosenthal
}

Published online: 11 January 2012

(C) The American Society for Experimental NeuroTherapeutics, Inc. 2012

\begin{abstract}
Neuromonitoring is an emerging field that aims to characterize real-time neurophysiology to tailor therapy for acute injuries of the central nervous system. While cardiac telemetry has been used for decades among patients requiring critical care of all kinds, neurophysiology and neurotelemetry has only recently emerged as a routine screening tool in comatose patients. The increasing utilization of electroencephalography in comatose patients is primarily due to the recognition of the common occurrence of nonconvulsive seizures among comatose patients, the development of quantitative measures to detect regional ischemia, and the appreciation of electroencephalography phenotypes that indicate prognosis after cardiac arrest. Other neuromonitoring tools, such as somatosensory evoked potentials have a complementary role, surveying the integrity of the neuroaxis as an indicator of prognosis or illness progression in both acute brain and spinal injuries.
\end{abstract}

Keywords Seizures · Critical care - Evoked potentials · Electroencephalography $\cdot$ Physiologic monitoring $\cdot$ Stroke . Cerebral hemorrhage $\cdot$ Traumatic brain injury $\cdot$ Subarachnoid hemorrhage

\section{Background}

Advances in continuous neuromonitoring have created an emerging environment in which state-of-the-art clinical care is paired with the ability to assess its effects in each individual patient. Certainly, the original form of neuromonitoring was the neurological examination, and tailored therapy based on clinical response has been the province of neurology for years.

\section{E. S. Rosenthal $(\square)$}

Department of Neurology, Division of Neurocritical Care and

Emergency Neurology, Massachusetts General Hospital,

55 Fruit St., Boston, MA 02114, USA

e-mail: erosenthal@partners.org
However, in the neurocritical care setting, the clinical examination is often clouded by coma, sedatives, toxic encephalopathy, or disorders of primary neurological modalities. As a result, the electroencephalogram (EEG) has increasingly been used to detect occult seizures, afford prognosis, and serve as a noninvasive and multiregional biomarker of ischemia or neuronal health. Similarly, evoked potentials have become increasingly important to provide a complete prognostic indicator and follow neurological function.

\section{Electroencephalography for Seizure Detection and Prognosis}

\section{Rationale and Indication}

EEG monitoring is critical for detecting seizures that may cause unexplained coma in patients requiring critical care. Only one quarter of patients are known to be in status epilepticus before it is documented by electroencephalography and subtle forms of status epilepticus are associated with longer delays to treatment and worse clinical outcomes $[1,2]$. In one series, seizure duration $\geq 1 \mathrm{~h}$ was associated with a tremendous increase in mortality compared with those lasting less than $1 \mathrm{~h}(2.7 \%$ vs 32\%) [3]. Nonconvulsive classification is more common when status is refractory ( $27 \%$ nonconvulsive at onset) vs. nonrefractory ( $2 \%$ nonconvulsive). As a result, patients with the least clinical evidence are at risk of the most refractory course, which itself is associated with month-long hospital stays, reduced clinical outcome at discharge, and complications, such as fever, pneumonia, hypotension, bacteremia, and blood transfusion [4]. The findings from EEG monitoring result directly affect clinical practice; within our own institution [5], providers adjusted seizure medications on the basis of EEG in more than half of the patients. 


\section{Technical Performance}

Limited montages, such as 4-channel sub-hairline recordings have been used to enable acute EEG acquisition when a technologist is unavailable [6], but limited sensitivity for seizure detection ( $70 \%)$ and for detecting spikes and periodic epileptiform discharges ( $40 \%$ ) have led to increasing performance of full-montage emergency EEG during offhour periods [7]. To afford rapid, full-surface EEG coverage during off-hour periods, certain centers have adopted the use of electrode templates [8] with or without the use of subdermal needle electrodes [9] to afford fast placement that does not require the technical skill needed to obtain adequate impedances with disc electrodes. Other institutions may include an intracortical depth electrode, given an experience in which depth seizures may be detected that were not diagnosed on the surface recording. At our institution, we currently use full-coverage scalp electrodes, and we have increasingly used conductive plastic electrodes using nonferromagnetic components; these electrodes are considered to have a magnetic resonance imaging compatible safety profile [10-12] and a computed tomography compatible by virtue of minimizing radiological artifact [13].

\section{Duration of Monitoring}

EEG studies have a role beyond spot monitoring because a short snapshot of monitoring often fails to fully characterize a patient's seizure propensity. Among one cohort of patients with intracerebral hemorrhage [14], 28\% of seizures were detected after the first $24 \mathrm{~h}$ of recording, suggesting that EEG has a role beyond spot monitoring. In a more inclusive cohort of 570 critically ill patients undergoing continuous EEG monitoring [15], the first electrographic seizure was discovered after the first day of monitoring in $12 \%$ of all patients and in $20 \%$ of comatose patients. As a result, when unexplained coma is present, or a patient is believed to have a high risk of seizure based on the underlying injury causing coma itself, $48 \mathrm{~h}$ of recording should be performed [16].

\section{Patient Selection}

Because of the intensive resource allocation needed to provide EEG monitoring in the neurocritical care environment, it is important to recognize patients at particularly high risk of subclinical seizures (Table 1).

In the setting of intracerebral hemorrhage, seizure rates up to $31 \%$ have been reported, more than half of which may have only nonconvulsive manifestation [14]. Almost half of seizures were detected beyond the first hour, whereas only $6 \%$ of seizures were detected after $48 \mathrm{~h}$ of monitoring. Seizures were more common with lobar hemorrhages $(23 \%)$ than in deep hemorrhages (11\%). Hemorrhage expansion and
Table 1 Prevalence of seizures among specific cohorts of patients referred for continuous EEG monitoring

\begin{tabular}{ll}
\hline Medical condition & Prevalence of seizures \\
\hline Intracerebral lobar hemorrhage & $23 \%$ \\
Intracerebral deep hemorrhage & $11 \%$ \\
Subarachnoid hemorrhage & $13-30 \%$ \\
Traumatic brain injury & $22-33 \%$ \\
Ischemic stroke & $6-10 \%$ \\
Hypoxic-ischemic encephalopathy & $10-33 \%$ \\
Sepsis & $32 \%$ \\
\hline
\end{tabular}

$\mathrm{EEG}=$ electroencephalogram

perihematomal edema resulting in midline shift have been associated with seizure, although the direction of causation is not established [14, 17]. To best allocate resources, guidelines have been focusing on recommending prolonged EEG for the detection of seizures in patients who have depressed mental status or a neurological examination out of proportion to the degree of documented brain injury [16].

In the setting of subarachnoid hemorrhage (SAH), patients with coma or unexplained neurological deterioration have a rate of nonconvulsive status epilepticus that exceeds $30 \%$ in highly selected patient populations, even despite prophylactic phenytoin administration [18], and which approaches $13 \%$ in our own hospital experience of patients with a clinical indication for EEG neuromonitoring (unpublished data). Risk factors for developing nonconvulsive status epilepticus include severe clinical presentation (Hunt and Hess grade IV or V), older age, and a focal pathologic lesions on computed tomographic imaging. In a separate cohort, $19 \%$ of patients undergoing EEG for SAH had documented seizures, almost all of which were nonconvulsive [19]. Because prophylactic anti-seizure medication is associated with worse outcome after SAH [20, 21], routine prophylaxis at our center is reserved for patients with high-grade $\mathrm{SAH}$ at risk of re-rupture from seizure prior to aneurysmal obliteration.

Among patients with traumatic brain injury (TBI), seizures have been reported in up to $33 \%$ of patients undergoing routine EEG following intensive care admission for TBI [22]. The first electrographic seizure was frequently detected more than 3 days after admission; contusions were a common risk for persistent seizures. In a separate cohort, more than half the $22 \%$ of patients with early post-traumatic seizures had nonconvulsive seizures alone, all despite prophylactic phenytoin administration [23]. Such nonconvulsive seizures produce prolonged elevation in ICP and signs of in vivo metabolic stress [24]. Conversely, when sudden intracranial hypertension occurs without other explanations, seizure is a frequent explanation [25]. In addition, seizures may be instigated or exacerbated by surgical evacuation of a subdural hematoma 
[26]. Post-traumatic seizures commonly result in long-term hippocampal atrophy ipsilateral to the seizure focus [27], with hippocampal neuronal loss as the pathologic phenotype [28]. Although investigators have reported a negligible seizure frequency when using the Lund concept method of preventing elevations in intracranial pressure, the success may be attributable to midazolam use as part of a bias to use sedation to prevent intracranial hypertension rather than the intracranial pressure control itself [29]. As a result, the literature supports the routine use of continuous monitoring for seizure detection in patients with TBI, despite the small amount of prevention afforded by 7 days of prophylactic phenytoin [30]. A secondary role of EEG in TBI is discrimination of seizures from paroxysmal autonomic instability with dystonia, both of which may be associated with elevated intracranial pressure [31, 32].

Hypoxic ischemic encephalopathy is also associated with a high rate of seizures, affecting 10 to $33 \%$ of patients following cardiac arrest $[33,34]$. Rewarming is a common time for seizures to occur, either because a clinical motoric component (e.g., myoclonic status epilepticus) is revealed after discontinuation of paralytics [34, 35], or because sedation or hypothermia suppressed or treated the electrographic activity [35]. In addition to detecting seizures, EEG demonstration of a reactive background rhythm or a continuous pattern on amplitude-integrated EEG had a high likelihood of good neurological prognosis, despite the presence of seizures [36-38]. Although myoclonic status may have a worse prognosis than nonconvulsive status epileptic in the setting of cardiac arrest treated with hypothermia, myoclonic status is not homogeneous and may have a worse prognosis when "reticular" rather than when associated with an EEG correlate [39]. More novel measures of EEG entropy are also able to demonstrate poor outcomes among patients with EEG waveforms of low complexity [40, 41].

Cerebral ischemia confers a lower risk of seizure. In the first $24 \mathrm{~h}$ after ischemic stroke, seizure occurs in $\leq 6.5 \%$ of patients [42], although two thirds of patients with seizures may develop status epilepticus, and rates may be $>10 \%$ among patients with severe strokes [43]. Status may occur in $>70 \%$ of patients with periodic discharges on EEG [42]. In addition to seizure detection, EEG has additional benefits in characterizing poor prognosis when there is background suppression, attenuation of fast frequencies [44], or when amplitude integrated values are low on quantitative EEG [45]. Good outcome may be seen in patients without significant slowing or change in background frequencies [44]. In addition, EEG may be used to demonstrate improvements in cerebral perfusion after induced hypertension or following volume challenges [46-48].

EEG may provide additional benefits in immunotherapy, and evaluation for an occult tumor should be a consideration in patients with refractory seizures when a syndrome of limbic encephalitis is present. Anti-Hu, anti-CV2/CRMP5 (collapsin response mediator protein-5), anti-ampiphysin, anti-alphaamino-3-hydroxy-5-methyl-4-isoxazolepropionic acid (AMPA), anti-N-methyl-d-aspartate receptor (NMDA), and anti-Ma2 limbic encephalitides are all associated with tumors or other malignancy. Evaluation for antibodies to small cell lung cancer (Hu, Ma2,CV2/CRMP5, AMPA, and ampiphysin), thymoma (collapsin response mediator protein 5 (CRMP5), AMPA, voltage-gated potassium channel (VGKC)), breast (AMPA), germ-cell testicular tumor (Ma2), or teratoma (NMDA) should be considered. Removal of the underlying tumor and immunotherapy with corticosteroids, plasma exchange, or intravenous immunoglobulins may often be helpful both for treating the seizures and their underlying paraneoplastic or autoimmune encephalitis. While anti-Hu is most commonly associated with seizures, Ma-2, NMDA, and AMPA-associated encephalitis and seizures are currently most treatable with immunotherapy, although AMPA receptor antibody syndrome often initially responds to immunotherapy, but may relapse [49]. Many similar conditions are observed to occur as nonparaneoplastic autoimmune encephalitides [50, 51], and may similarly respond to steroids or immunotherapy.

Poorly appreciated patient populations at risk for seizures are those with primary psychiatric diseases or dementia. Such patients often have periodic patterns or triphasic wave patterns in association with background slowing [52].

Metabolic encephalopathy itself is a high risk for seizures. A prospective review of 681 patients undergoing emergency EEG at a 700-bed tertiary hospital in Brazil revealed that studies performed for seizure, hepatic encephalopathy, or impaired consciousness led to the following as the most common final diagnoses: liver disease $17.5 \%$, stroke (15.4\%), metabolic disorders $(9.9 \%)$, central nervous system infection $(7.5 \%)$, and prior epilepsy (7.2\%). Hepatic encephalopathy is commonly associated with a metabolic etiology, but seizures may contribute as well; approximately $15 \%$ of patients with hepatic encephalopathy have epileptiform abnormalities on EEG, more than half of which involve frank electrographic seizures [53]. Neurological complications more than double length of stay in the medical intensive care environment, with sepsis a particularly potent cause of epileptic encephalopathy. Among a cohort of patients monitored by EEG in a medical intensive care unit, the rate of electrographic seizures or periodic discharges was more than triple among patients with sepsis compared with those without (32\%vs $9 \%)$, a characteristic of independent risk that was more significant than a history of epilepsy or clinical seizures suspected prior to the initiation of monitoring [54]. A number of medical diseases causing mental status changes are increasingly being appreciated for a direct neurological mechanism by which seizures mediate a previously regarded "medical" encephalopathy, such as that in patients with thrombotic thrombocytopenic purpura [55]. 
Considering mental status itself across multiple disease types, the absence of coma does not exclude the presence of seizure. In a convenience sample of patients with head trauma, spontaneous hemorrhage, or brain tumor within a neurosurgical unit, 28 of 158 patients had nonconvulsive status epilepticus on EEG, but $40 \%$ of the patients with nonconvulsive status epilepticus had a Glasgow Coma Scale score $\geq 9$ [56]. Similarly, in patients with sepsis found to have seizures, the presence of coma was not associated with a significant difference in the prevalence of electrographic seizures or periodic discharges $(24 \%$ among comatose patients and $20 \%$ among noncomatose patients) [54].

A future role exists for serologic or genetic biomarkers predicting seizure risk; genetic predisposition to status epilepticus has been suggested in twin studies, however, currently no role exists for routine screening for susceptibility [57].

\section{Significance of EEG Patterns on the Ictal-Interictal Continuum and of Cortical Spreading Depression}

\section{Periodic Discharges}

Periodic lateralized epileptiform discharges (PLEDs) (Fig. 1) or lateralized periodic discharges are electrographic injury patterns often present on surface EEG during an acute presentation from ischemic stroke, intracerebral hemorrhage, subdural hematoma, subarachnoid hemorrhage, malignancy, hypoxic-ischemic encephalopathy, encephalitis, posterior reversible encephalopathy syndrome, or other forms of acute brain injury, or one of these structural causes in association with superimposed metabolic encephalopathy [58]. The majority of patients with PLEDs on initial EEG either had a prior seizure or will go on to have future clinical seizures [59]. Accordingly, these patterns are often treated with antiseizure medication. However, they are not routinely suppressed with continuous infusions given that their significance may vary from patient to patient. Certain patterns of PLEDs with intervening rhythmic activity, typically of low amplitude, may be associated with greater risk of seizure [60, 61].

Generalized periodic epileptiform discharges (GPEDs) confer a poor overall prognosis, although it may vary based on etiology. Among a series of patients with GPEDs, including post-hypoxic myoclonic encephalopathy, prion diseases, and encephalitis, approximately $60 \%$ died after 2 months; there was a $50 \%$ mortality rate for patients with status epilepticus, PLEDs, or continuous delta activity commonly related to underlying advanced liver disease [62].

\section{Status Epilepticus}

Seizure risk is particularly high after termination of convulsive status epilepticus; approximately $48 \%$ of patients have persistent electrographic seizures, either with subtle or no ostensible observable correlation. After the convulsive status subsides, $30 \%$ of those patients with subtle or nonconvulsive seizures develop nonconvulsive status epilepticus [63]. Status epilepticus itself results in $21 \%$ mortality, with age (odds ratio [OR], 1.1) and acute symptomatic cause for the seizure activity $(\mathrm{OR}, 6.0)$ found to be multivariate predictors of mortality. Morbidity is similarly high, with $23 \%$ of nonfatal episodes involving a functional decline between admission and discharge. A length of stay exceeding the median (OR, 1.04) and acute symptomatic seizure (OR, 3.9) are risk factors independently associated with functional decline during hospitalization [64].

The presence of these seizures or of nonconvulsive status epilepticus after convulsive status epilepticus has terminated is associated with higher mortality $(30 \%$ and $50 \%$, respectively) than those without either pattern (approximately $13 \%)$. Multivariate regression revealed a doubling in the odds of mortality among these patients who transitioned to nonconvulsive status epilepticus and an independent doubling in the odds of mortality among patients $>60$ years of age [63].

\section{Stimulus-Induced Patterns}

Certain seizure-like patterns may appear that are particularly linked to stimulation. These stimulus-induced rhythmic, periodic, or ictal discharges (SIRPIDs) have been observed in $22 \%$ of patients undergoing continuous EEG monitoring during critical illness. Associated status epilepticus is particularly common when these SIRPIDs are focal or particularly ictal in appearance [65]. It is not known whether treating these stimulus-related patterns or decreasing noise and clinical stimulation has an impact on clinical outcome.

Triphasic Waves and Discharges with Triphasic Morphology

Triphasic waves were traditionally described as an electrographic phenomenon consisting of periodic sharp waves with a triphasic "check mark" waveform in association with metabolic encephalopathies from hepatic or renal etiology [66], or alternatively after anoxic-ischemic encephalopathy, Creutzfeldt-Jakob disease, or toxicity from lithium, valproate, or baclofen toxicity [67-71]. Other conditions causing triphasic waves include brainstemdiencephalic tumors or infarcts, or alternatively diffuse or multifocal encephalopathy from vascular white matter disease or lymphoma [72]. Nonconvulsive status, however, may masquerade as a triphasic encephalopathy (e.g., in association with cephalosporin overdose in the setting of renal dysfunction) [73]. Husain et al. [74] sought to 
Fig. 1 Examples of common electrographic patterns, displayed at $30 \mathrm{~mm} / \mathrm{s}$ time base, $7 \mathrm{uV} / \mathrm{mm}$ sensitivity, $1-\mathrm{Hz}$ low frequency filter, and $70-\mathrm{Hz}$ high frequency filter. (a) A patient with recent subarachnoid hemorrhage following anterior communicating artery aneurysm clipping. Electroencephalogram (EEG) monitoring revealed frequent runs of left anterior lateralized rhythmic delta activity with admixed sharp components (LA-LRDA $+\mathrm{s}$ ) at $1-1.5 \mathrm{~Hz}$; time marker denotes additional epileptiform sharp components. (b) A patient with a newly diagnosed right temporal brain mass, presumed metastatic from hepatocellular carcinoma. The routine EEG showed right lateralized periodic discharges with admixed fast activity at approximately $1.5 \mathrm{~Hz}$; time marker notes an associated period of relative background suppression. (c) The same patient depicted in (b), but with a subsequent period of chewing movements (note movement artifact), increased bilateral representation of the activity, and increased frequency. This discrete period of evolution is consistent with a seizure with a subtle clinical correlation. Shortly after EEG placement, the patient was found to be having seizures on an average of every 15 minutes

\section{a}

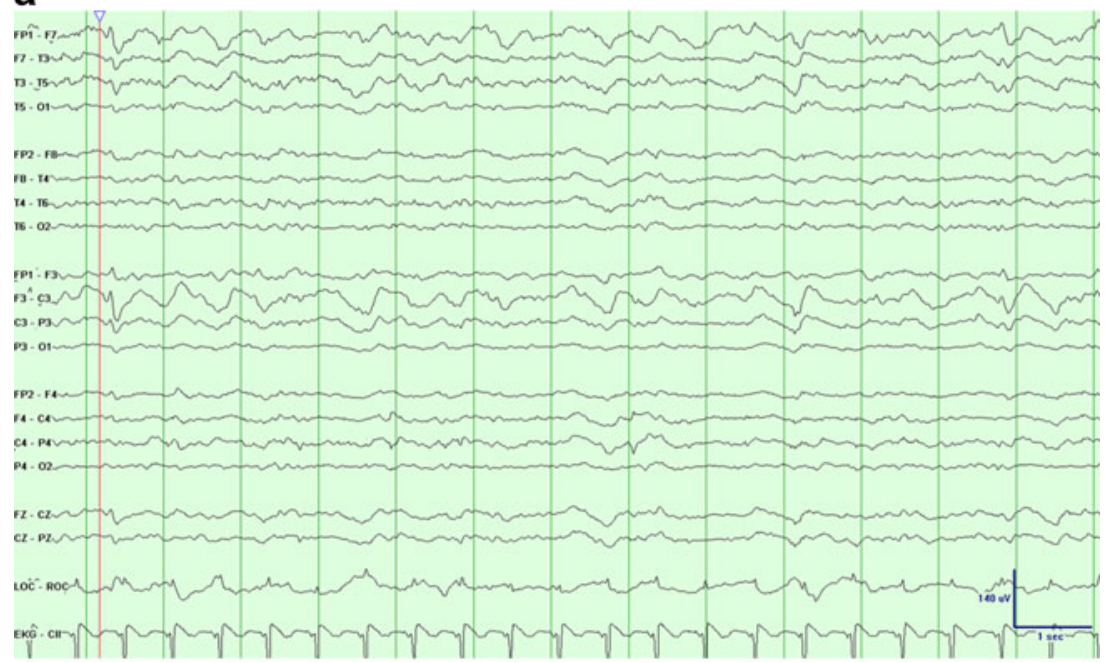

b

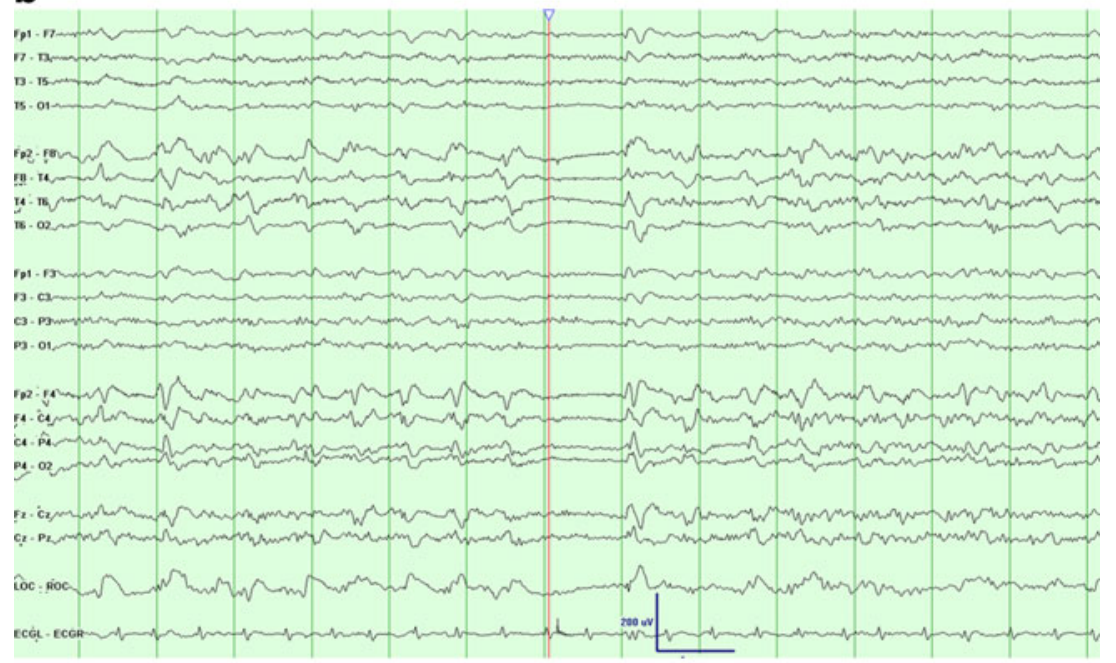

C

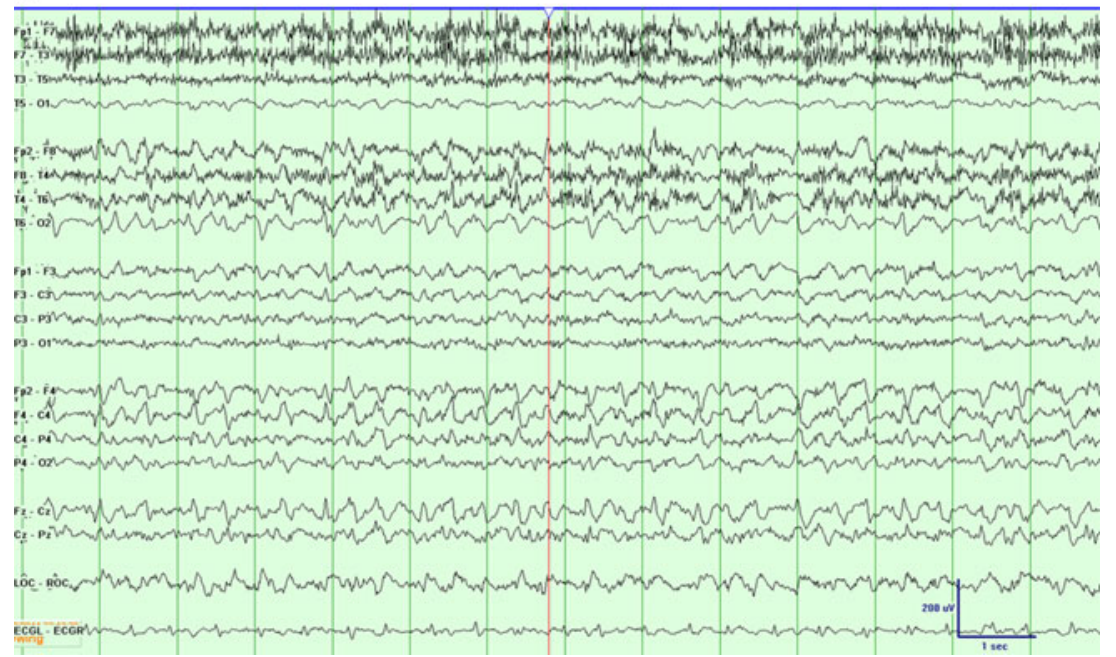

discriminate which GPED patterns were most likely to be associated with status epilepticus; GPEDs with high amplitude and longer duration, and those which preserved
inter-GPED amplitude were more commonly associated with status epilepticus; however, no cut-point had sufficient receiver-operator characteristics to permit discrimination. 
Accordingly, we often challenge patients with a benzodiazepine trial to assess clinical response, or more rarely sustained improvement in the EEG background rhythm to discriminate between ictal and metabolic encephalopathy associated with triphasic waves.

\section{Cortical Spreading Depression}

Cortical spreading depression is an electrical wave of depolarization that spreads over the cortex and may increase cortical metabolic demands, causing deleterious effects in stroke, subarachnoid hemorrhage, intracerebral hemorrhage, TBI, and other acute neurological diseases. Methods using invasive electrocorticography and laser Doppler flowmetry have characterized cortical spreading depression (CSD) as causing ischemia related to low-frequency vascular fluctuations with a frequency less than $0.1 \mathrm{~Hz}$ and a propagation rate over the cortex of 2 to $5 \mathrm{~mm} / \mathrm{min}$ [75].

The implication of the CSD is a significant metabolic stress and risk of ischemic infarction. In rodent models of experimental ischemia, CSD resulted in reduced cerebral blood flow [76] and increased infarct volume [77]. In human studies, cortical spreading depression has been associated with metabolic stress and injury, as measured by elevation in glutamate and lactate/pyruvate ratio, as well as low brain tissue oxygenation [78]. Its effects are not clearly mediated through or detected by rises in intracranial pressure [79], and thus CSD may result in secondary injury independent of intracranial pressure. Accordingly, CSD may represent either a therapeutic target in itself or a biomarker capable of guiding cerebral perfusion pressure and temperature management. In SAH, CSD provided excellent detection of subsequent delayed ischemic neurological deficits (positive predictive value, $86 \%$; negative predictive value, $100 \%$ ). The events of neurological decline were closely time-linked to $\mathrm{CSD}$, providing biologic plausibility for their role as more than an epiphenomenon [80]. Invasive measures of brain tissue oxygenation showed that waves of CSD following SAH resulted in significant stress on the balance of brain oxygen supply and demand, causing waves of brain tissue hypoxia followed by rebound hyperoxia. This biphasic pattern transitioned to monophasic hypoxia once a delayed neurological ischemic deficit has occurred, suggesting that CSD can cause irreversible injury when occurring in vulnerable tissue [81].

While invasive corticography from an intracranial electrode strip placed on the cortical surface is the most robust method to detect CSD, other noninvasive methods, such as near-infrared spectroscopy may offer a noninvasive surrogate for CSD by monitoring its effect on cerebral blood flow [82-85].

\section{Treatment of Patterns on the Ictal-Interictal Continuum}

\author{
Factors Associated with Successful Treatment
}

When status epilepticus is present, there is a clear need for prompt and comprehensive treatment. Most clinicians treat with more than 1 nonanesthetic medication before inducing coma or burst suppression to treat status epilepticus, and withhold anesthetic seizure medications in the setting of nonconvulsive status epilepticus [86]. This approach is based on the concept that complex partial status epilepticus may result in less toxicity and morbidity than generalized convulsive status epilepticus [87]. In 1 study, adherence to a dedicated protocol for status epilepticus occurred in only $38 \%$ of patients, although adequate first-line therapy was associated with an almost sevenfold likelihood of seizure termination and reduced length of stay [88].

\section{Hierarchy of Therapies for Managing Status Epilepticus}

The management of status epilepticus is approached in stepwise fashion. Airway and circulatory compromise are emergently managed. In a second phase, thiamine and glucose along with benzodiazepines are given.

Acute benzodiazepine use was the treatment of choice in the Veterans Affairs Status Epilepticus Cooperative Study Group, which evaluated different strategies in a randomized and double-blind clinical trial [1]. Intravenous lorazepam yielded successful seizure control in approximately $65 \%$ of patients, compared with rates of control of $58 \%$ or less among the 3 other treatments (phenobarbital, diazepam, and phenytoin, or phenytoin alone; these are listed in decreasing likelihood of success). In addition, lorazepam has pharmacokinetic properties that favor rapid central nervous system penetration without rapid redistribution. Fosphenytoin requires approximately 10 minutes before being metabolized to an active medication. Phenytoin itself must be administered slowly, given the risk of dysrhythmia or bradycardia. Patients with epilepsy and frequent admissions for status epilepticus may benefit from intranasal midazolam, which may be given at home prior to arrival at the hospital; although intravenous diazepam has a shortened time to seizure cessation, nasal midazolam may be more quickly administered [89]. A similar role exists for rectal formulations of diazepam or sublingual administration of lorazepam.

A second-line anti-seizure medication should be requested at the same time as the initial therapy is selected, simply given that more than a third of patients presenting in status are refractory to benzodiazepines [1]. Phenytoin or valproic acid are common second-line therapies, both due to easy route of intravenous loading and due to their clinical efficacy without respiratory depression. Open-label administration of intravenous 
levetiracetam has become a more frequently used second-line anti-seizure medication, aborting status epilepticus in up to $70 \%$ of benzodiazepine-refractory patients [90]. Separate investigations have also supported the usefulness of intravenous lacosamide in open-label experiences. Status epilepticus was aborted in 3 of 5 uses of intravenous lacosamide as a first- or second-line medication, in 11 of 19 as a third-line medication, and in 3 of 15 thereafter; whereas 56\% required an additional medication [91]. Similarly promising results were seen with the use of topiramate for patients with refractory generalized and complex partial status epilepticus, although an intravenous formulation is not yet available [92, 93].

Intravenous anesthetic doses of medications, such as pentobarbital, propofol, and midazolam are recommended as third-line therapies after intravenous lorazepam and an intravenous seizure medication. Placement of arterial blood pressure monitoring or central venous access for administration of vasopressors may be necessary, given the hypotension associated with these therapies [94]. In addition, nonbarbiturate therapy may be preferred due to a shorter duration of respiratory depression and mechanical ventilation [94].

\section{Gauging the Response to Treatment}

Gauging the response to treatment therapy is best accomplished with EEG at the bedside, even for convulsive status epilepticus, given its high likelihood of transforming to subtle or nonconvulsive status epilepticus [63]. EEG may also be used to gauge success at achieving electrographic suppression or burst suppression, which has been associated with decreased likelihood of relapsed status epilepticus following barbiturate therapy [95] with fewer breakthrough seizures [96].

Neuron-specific enolase [97-99] and diffusion-weighted imaging [100-102] have been promulgated as biomarkers, which may detect nonconvulsive or complex partial status epilepticus; however, neither has been shown to be a reliable marker for anti-seizure medication titration in status epilepticus or in determining which periodic or stimulus-induced patterns on the ictal-interictal continuum should be treated. Cerebral microdialysis for the detection of elevated lactate or lactate:pyruvate ratio may help detect subtle seizures that surface EEG may miss [103]. Brain tissue oxygenation, however, has not been found to have a meaningful response to seizures, limiting its use for clinical seizure detection [104].

Patterns on the ictal-interictal continuum, such as PLEDs and GPEDs, are of uncertain significance, resulting in heterogeneous practice patterns. Practitioners often use adjunctive tools, such as positron emission tomography or perfusion magnetic resonance imaging to assess whether these patterns effect a change in cerebral blood flow. Patterns associated with a temporal change in intracranial pressure or elevations in lactate, lactate:pyruvate ratio, or glycerol on cerebral microdialysis are other tools suggested to reveal pathologic changes related to periodic discharges or SIRPID [105]. Nevertheless, these anecdotal observations have not been validated. The clinical significance of these intermediate patterns may be best assessed by clinical monitoring following treatment with a benzodiazepine challenge or institution of a therapeutic dose of an anti-seizure medication. A caveat is that the EEG and acute behavior will often show suppression to benzodiazepines, such that a durable clinical benefit is a more reasonable measure of treatment success. At the minimum, most periodic patterns should be treated with anti-seizure medication of some type, given the high frequency in which they are associated with subsequent seizures [87].

\section{Titration of Barbiturates for Intracranial Hypertension}

An additional role for EEG in guiding therapy is in the management of elevated intracranial pressure. Although still controversial, due to the effects of hypotension and infection [106], barbiturate sedation should be performed in conjunction with EEG to minimize the amount of medication by treating only until burst suppression has been obtained.

\section{Quantitative EEG for Ischemic Monitoring}

\section{Historical Role}

Although continuous EEG has an important role in the detection of seizures, it has a historical and re-emerging role in the detection and monitoring of cerebral ischemia. The EEG may reveal neurological decline and recovery before the clinical response. At cerebral blood flow levels below 20 $\mathrm{ml} / 100 \mathrm{~g} / \mathrm{min}$, there is progressive loss of alpha and beta EEG frequencies and slowing of the dominant background rhythm [107].

\section{Quantitative Tools and Technical Methods}

Quantitative EEG is not a single tool, but rather a series of instruments that depict mathematical properties of the EEG over a duration of time (Fig. 2).

EEG power spectral analysis trends show the voltage (amplitude) contributed to the EEG by each frequency over a duration of time. They are computed using Fast-FourierTransformation analysis of the EEG, followed by assessment of amplitude in each frequency band. Frequency is listed on the vertical access, and color typically provides a measure of amplitude, which may be visually represented as the square-root of voltage to permit a useful dynamic range among high-amplitude waves of low frequency and lowamplitude waves in the high frequency spectra (e.g., beta activity). Often, measures are calculated for specific regions 


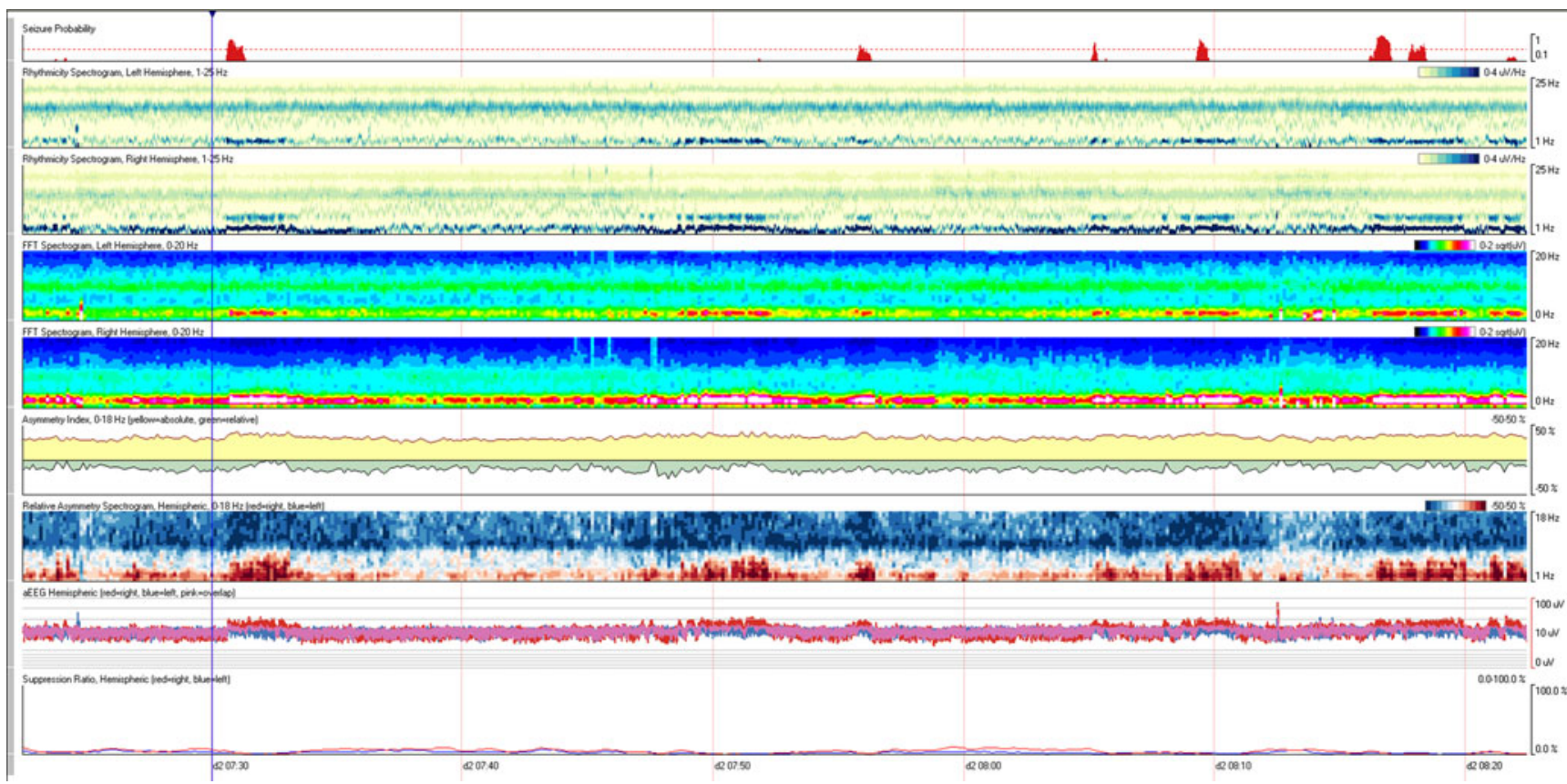

Fig. 2 Example of a quantitative electroencephalogram (EEG) panel in a patient with intermittent rhythmic delta activity, showing measures including seizure probability, computed frequency power spectral array for each hemisphere, relative and absolute asymmetry indices, relative asymmetry spectral array, amplitude-integrated EEG, and a suppression ratio in the brain (e.g., left and right hemisphere, four quadrants, or to approximate specific vascular territories when monitoring for ischemia). Spectral analysis from each hemisphere may also be compared to provide an asymmetry spectrogram to assess the difference in amplitude between hemispheres for each frequency.

Fast-Fourier-Transformation power trends assess either the total power in a certain band of frequencies (e.g., total alpha power [108]) or the ratio of power (voltage) in 2 specific frequency bands (e.g., power in the alpha range of frequencies $v s$ power in the delta range of frequencies, i.e., "alpha-delta ratio") [109]. Ratios may adjust for artifacts, which increase power in multiple frequency ranges and magnify the effect of transitions from faster alpha frequencies within health brain tissue to slower delta rhythms following ischemia, transitions to sleep, or in the intra-ictal and post-ictal states. This data may be averaged over a duration of time or depicted without averaging to permit visual inspection of alpha power variability, for example [110].

Amplitude-integrated EEG is another quantitative tool that provides information regarding the amplitude and variation of cerebral activity during a short time constant to demonstrate fluctuations in the amplitude of cerebral activity. Thickness of the tracing is proportional to the moment-to-moment amplitude variability, while the overall magnitude of the tracing reflects the voltage amplitude of the EEG at each period of time. For example, burst-suppression would result in a thick tracing due to alternating bursts and suppressions, whereas amplitude increases during seizure may result in increase in the overall magnitude of the tracing [111]. Electrodecremental seizures or decrease in EEG amplitudes during anesthesia may result in the opposite effect.

Asymmetry indexes are computed based on a comparison of frequencies between hemispheres. If one hemisphere develops greater voltages than the contralateral side for any single frequency, this change will increase the degree of asymmetry. Both absolute measures and relative measures may be trended to help detect the total burden of asymmetry for all frequencies (absolute) and to depict the relative balance of asymmetry in each frequency band. For example, if there is delta activity of higher amplitude on the left than the right, then a subsequent increase in beta activity on the right will further increase the absolute asymmetry index, but move the relative asymmetry index toward or past zero. These trends may hide which frequencies are the asymmetric ones, a tool highlighted by the relative spectrogram, but they are useful in providing numerical measures and overall quantitative values of asymmetry, which can be numerically trended.

\section{Usefulness in Ischemic Stroke Monitoring}

In acute ischemic stroke, quantitative EEG provides a good assessment of stroke size and severity. Measuring the rate of change in average delta power yields a modest correlation with volume of initial mean transit time $(r=0.79 ; p<0.01)$, follow-up diffusion-weighted imaging volume $(0.62 ; p<$ 0.05 ), and 30-day National Institutes of Health Stroke Scale (NIHSS) score $(r=0.80 ; p<0.01)$ [112]. Subsequent 
quantitative EEG measures (subacute delta-alpha power ratio and relative alpha power) strongly correlated with 30-day NIHSS $(r=0.91 ; p<0.001$ and $r=0.82 ; p<0.01$, respectively) [113]. A similarly high correlation between quantitative EEG measures of ischemia was found by other investigators using a brain symmetry index [114]. As a measure of stroke severity and recovery, advanced quantitative EEG measures of functional cerebral connectivity have been recently introduced as a novel metric to assess stroke severity and monitor recovery $[115,116]$.

Usefulness in Vasospasm Monitoring after Aneurysmal Subarachnoid Hemorrhage

EEG has become widely adopted for the detection of SAHrelated vasospasm. Initial experience with quantitative EEG suggested that total power provides high sensitivity exceeding $90 \%$ for ischemia following subarachnoid hemorrhage [108]. Quantitative EEG variables, such as relative alpha variability trend of total power and post-stimulation alphadelta ratio (ADR) may be used as an early warning system to detect slowing and loss of fast activity, reflective of focal ischemia in an early timeframe before it is clinically apparent in SAH patients with predominantly good- or poor-grade SAH $[109,110]$. In SAH patients with depressed level of arousal or coma, such as Hunt-Hess grade IV or V patients, a decline in ADR during periods of arousal has been associated with the development of delayed cerebral ischemia. Of a 34-patient cohort, 9 who developed DCI had a median ADR decrease of $24 \%$, and ADR decrement more than $10 \%$ from baseline during 6 consecutive measures was $100 \%$ sensitive and $76 \%$ specific for delayed cerebral ischemia. Similarly, a single measurement showing more than $50 \%$ decrement from baseline had good sensitivity $(89 \%)$ and specificity (84\%) [109]. Another EEG measure, relative alpha variability, has been shown to have excellent sensitivity for detecting angiographic vasospasm by using its qualitative scale. More invasive measures using ADR derived from intracortical EEG have been demonstrated in a small cohort to provide additional value over surface EEG measures [117].

Thus, EEG measures provide excellent accuracy for detecting a clinically meaningful outcome. In comparison, TCD sensitivity for DCI is only $63 \%$ [118]. For certain vascular territories, the use of TCD is a struggle to even accurately detect a radiological surrogate, such as an angiographic vasospasm [119]. Even for angiographic surrogates, TCD struggles to detect angiographic vasospasm in the anterior cerebral artery (ACA) vessels and the posterior cerebral artery (PCA) vessels. Sensitivity and specificity for moderate-to-severe angiographic ACA vasospasm is only $35 \%$ and $65 \%$, respectively, for TCD mean velocity of $120 \mathrm{~cm} / \mathrm{sec}$. Receiver operator curve characteristics do not permit a significantly higher specificity at more stringent
TCD thresholds. For posterior cerebral artery vasospasm, sensitivity is $75 \%$ and specificity is $69 \%$. Vasospasm is often missed in vessels that could not be insonated (e.g., pericallosal anterior cerebral artery), in situations in which other anatomic factors prevailed, or in relation to operator inexperience or error. Similarly, poor TCD sensitivity for detecting DCI has been noted in other populations, particularly in patients with aneurysms of the internal carotid artery $(66.6 \%)$ or anterior communicating artery (40.6\%) [120]. Although these quantitative EEG tools appear robust and promising for the detection of patients at risk for DCI, it is not clear if their response to treatment can afford a biomarker for tailoring subsequent hemodynamic augmentation.

\section{SSEPs for Prognosis and Monitoring}

\section{SSEP in Traumatic Brain Injury}

Another robust neurophysiologic tool is the use of evoked cortical potentials. Most useful in the neurocritical care setting are median nerve somatosensory evoked potentials (SSEPs), which interrogate the intact connectivity of peripheral sensory nerves to cortical projections. Motor evoked potentials, visual evoked potentials, and brainstem evoked potentials provide an analogous interrogation of other pathways. SSEPs are assessed as normal, abnormal (increased latency or reduced amplitude), or absent on each side, providing the basis for a 6-point ordinal scale in which both hemispheres are considered. When bilaterally absent or alternatively normal following TBI, SSEPs may help detect patients with poor or good prognosis [121]. For example, normal SSEPs (grade 1) after TBI are associated with a $57 \%$ chance of good recovery, whereas bilaterally absent SSEPs (grade 6) are associated with only a $1 \%$ chance of functional recovery [122]. Unfortunately, intermediate test results are less helpful. Further studies have shown that at day 3 , these studies may predict cognitive functions, such as attention, concentration, processing speed, and working memory [123]. In addition, repeated SSEP measures may also help detect patients with brainstem herniation due to interruption of these functional connections [124], or with cerebral ischemia correlating with jugular bulb evidence of reduced oxygen content [125]. Changes in median SSEP may precede the rise in intracranial pressure $30 \%$ of the time [126].

\section{SSEP in Spinal Cord Injury}

Similarly, SSEPs and motor evoked potentials may provide prognostic information indicative of recovering ambulation, hand, and bladder function after spinal cord injury [127] or 
as a supplement to clinical testing to predict functional outcome measured by the Barthel Index [128]. These SSEP findings are even more robust when they fail to improve with time [129]. Similarly, neurophysiologic sympathetic skin recordings can provide information regarding the extent and level of lesions of the spinal sympathetic nervous system [127]. In general, evoked potentials are less confounded by sedation or hypothermia than is EEG [130].

\section{SSEP in Hypoxic-Ischemic Encephalopathy}

Following cardiac arrest, SSEPs have a better capacity to identify patients with poor outcome than to predict good outcome. Among 1136 patients with hypoxic-ischemic encephalopathy after cardiac arrest, absent SSEPs were associated with no patients awakening from coma [122], particularly when combined with absent EEG reactivity [131], although withdrawal of care may have biased such results. Normal SSEPs had less predictive capacity; in the same cohort, only $52 \%$ of patients with normal SSEPs awakened from coma [122]. The ability of absent SSEPs to detect patients at risk for poor neurological outcome appear to be robust even among patients undergoing therapeutic hypothermia following arrest [132], although withdrawal of care may bias this and other retrospective experiences.

\section{Conclusion}

In summary, a high percentage of patients requiring neurocritical care develop nonconvulsive seizures associated with poor outcome. These seizures would be undetectable without EEG neuromonitoring. In addition to information regarding subclinical seizures, EEG provides: prognostic information following cardiac arrest; characterization of seizures, which may be associated with intracerebral hemorrhage expansion and midline shift; prediction of ischemia from vasospasm after $\mathrm{SAH}$; and guidance of seizure management and barbiturate therapy for refractory intracranial hypertension. One difficult byproduct of expanded EEG use has been the unclear management of intermediate patterns, such as periodic discharges, SIRPIDs, or triphasic waves, which are currently best managed with electroclinical correlation following provision of medications, such as benzodiazepines or other anti-seizure medications. SSEP is a complimentary technology, which is robust, despite sedation and hypothermia, providing prognostic information after cardiac arrest, TBI, and spinal cord injury. After TBI, serial SSEP measures may promote early detection of intracranial hypertension or herniation. As a result of these multifaceted roles, EEG and SSEP have achieved a significant role in neurocritical care.
Required Author Forms Disclosure forms provided by the authors are available with the online version of this article.

\section{References}

1. Treiman DM, Meyers PD, Walton NY, et al. A comparison of four treatments for generalized convulsive status epilepticus. Veterans Affairs Status Epilepticus Cooperative Study Group. NEJM 1998;339:792-798.

2. Drislane FW, Lopez MR, Blum AS, Schomer DL. Detection and treatment of refractory status epilepticus in the intensive care unit. J Clin Neurophysiol 2008;25:181-186.

3. Towne AR, Pellock JM, Ko D, DeLorenzo RJ. Determinants of mortality in status epilepticus. Epilepsia 1994;35:27-34.

4. Mayer SA, Claassen J, Lokin J, Mendelsohn F, Dennis LJ, Fitzsimmons BF. Refractory status epilepticus: frequency, risk factors, and impact on outcome. Arch Neurol 2002;59:205-210.

5. Kilbride RD, Costello DJ, Chiappa KH. How seizure detection by continuous electroencephalographic monitoring affects the prescribing of antiepileptic medications. Arch Neurol 2009;66:723-728.

6. Bubrick EJ, Bromfield EB, Dworetzky BA. Utilization of belowthe-hairline EEG in detecting subclinical seizures. Clin EEG Neurosci 2010;41:15-18.

7. Young GB, Sharpe MD, Savard M, Al Thenayan E, Norton L, Davies-Schinkel C. Seizure detection with a commercially available bedside EEG monitor and the subhairline montage. Neurocrit Care 2009;11:411-416.

8. Jordan KG, Schneider AL. Emergency ("stat") EEG in the era of nonconvulsive status epilepticus. Am J Electroneurodiagnostic Technol 2009;49:94-104.

9. Schneider AL. Subdermal needle electrodes: An option for emergency ("stat") EEGs. Am J Electroneurodiagnostic Technol 2006;46:363-368.

10. Vulliemoz S, Perrig S, Pellise D, et al. Imaging compatible electrodes for continuous electroencephalogram monitoring in the intensive care unit. J Clin Neurophysiol 2009;26:236-243.

11. Mirsattari SM, Davies-Schinkel C, Young GB, Sharpe MD, Ives JR, Lee DH. Usefulness of a 1.5 T MRI-compatible eeg electrode system for routine use in the intensive care unit of a tertiary care hospital. Epilepsy Res 2009;84:28-32.

12. Mirsattari SM, Lee DH, Jones D, Bihari F, Ives JR. MRI compatible EEG electrode system for routine use in the epilepsy monitoring unit and intensive care unit. Clin Neurophysiol 2004; $115: 2175-2180$.

13. Das RR, Lucey BP, Chou SH, et al. The utility of conductive plastic electrodes in prolonged icu EEG monitoring. Neurocritical care 2009;10:368-372.

14. Claassen J, Jette N, Chum F, et al. Electrographic seizures and periodic discharges after intracerebral hemorrhage. Neurology 2007;69:1356-1365.

15. Claassen J, Mayer SA, Kowalski RG, Emerson RG, Hirsch LJ. Detection of electrographic seizures with continuous EEG monitoring in critically ill patients. Neurology 2004;62:1743-1748.

16. Morgenstern LB, Hemphill JC 3rd, Anderson C, et al. Guidelines for the management of spontaneous intracerebral hemorrhage: a guideline for healthcare professionals from the American Heart Association/American Stroke Association. Stroke 2010;41:21082129.

17. Vespa PM, O'Phelan K, Shah M, et al. Acute seizures after intracerebral hemorrhage: A factor in progressive midline shift and outcome. Neurology 2003;60:1441-1446.

18. Dennis LJ, Claassen J, Hirsch LJ, Emerson RG, Connolly ES, Mayer SA. Nonconvulsive status epilepticus after subarachnoid hemorrhage. Neurosurgery 2002;51:1136-1144. 
19. Claassen J, Mayer SA, Hirsch LJ. Continuous EEG monitoring in patients with subarachnoid hemorrhage. J Clin Neurophysiol 2005;22:92-98.

20. Rosengart AJ, Huo JD, Tolentino J, et al. Outcome in patients with subarachnoid hemorrhage treated with antiepileptic drugs. Journal of Neurosurgery 2007;107:253-260.

21. Naidech AM, Kreiter KT, Janjua N, et al. Phenytoin exposure is associated with functional and cognitive disability after subarachnoid hemorrhage. Stroke 2005;36:583-587.

22. Ronne-Engstrom E, Winkler T. Continuous EEG monitoring in patients with traumatic brain injury reveals a high incidence of epileptiform activity. Acta Neurol Scand 2006;114:47-53.

23. Vespa PM, Nuwer MR, Nenov V, et al. Increased incidence and impact of nonconvulsive and convulsive seizures after traumatic brain injury as detected by continuous electroencephalographic monitoring. J Neurosurg 1999;91:750-760.

24. Vespa PM, Miller C, McArthur D, et al. Nonconvulsive electrographic seizures after traumatic brain injury result in a delayed, prolonged increase in intracranial pressure and metabolic crisis. Crit Care Med 2007;35:2830-2836.

25. Marienne JP, Robert G, Bagnat E. Post-traumatic acute rise of ICP related to subclinical epileptic seizures. Acta Neurochir 1979;28:89-92.

26. Rabinstein AA, Chung SY, Rudzinski LA, Lanzino G. Seizures after evacuation of subdural hematomas: Incidence, risk factors, and functional impact. J Neurosurg 2010;112:455460.

27. Vespa PM, McArthur DL, Xu Y, et al. Nonconvulsive seizures after traumatic brain injury are associated with hippocampal atrophy. Neurology 2010;75:792-798.

28. Swartz BE, Houser CR, Tomiyasu U, et al. Hippocampal cell loss in posttraumatic human epilepsy. Epilepsia 2006;47:13731382.

29. Olivecrona M, Zetterlund B, Rodling-Wahlstrom M, Naredi S, Koskinen LO. Absence of electroencephalographic seizure activity in patients treated for head injury with an intracranial pressuretargeted therapy. J Neurosurg 2009;110:300-305.

30. Chang BS, Lowenstein DH. Practice parameter: Antiepileptic drug prophylaxis in severe traumatic brain injury: Report of the Quality Standards Subcommittee of the American Academy of Neurology. Neurology 2003;60:10-16.

31. Boeve BF, Wijdicks EF, Benarroch EE, Schmidt KD. Paroxysmal sympathetic storms ("diencephalic seizures") after severe diffuse axonal head injury. Mayo Clin Proc 1998;73:148-152.

32. Blackman JA, Patrick PD, Buck ML, Rust RS Jr. Paroxysmal autonomic instability with dystonia after brain injury. Arch Neurol 2004;61:321-328.

33. Legriel S, Bruneel F, Sediri H, et al. Early EEG monitoring for detecting postanoxic status epilepticus during therapeutic hypothermia: a pilot study. Neurocrit Care 2009;11:338-344.

34. Rossetti AO, Logroscino G, Liaudet L, et al. Status epilepticus: An independent outcome predictor after cerebral anoxia. Neurology 2007;69:255-260

35. Abend NS, Topjian A, Ichord R, et al. Electroencephalographic monitoring during hypothermia after pediatric cardiac arrest. Neurology 2009;72:1931-1940.

36. Rossetti AO, Urbano LA, Delodder F, Kaplan PW, Oddo M. Prognostic value of continuous EEG monitoring during therapeutic hypothermia after cardiac arrest. Crit Care 2010;14:R173.

37. Rundgren M, Rosen I, Friberg H. Amplitude-integrated eeg (aEEG) predicts outcome after cardiac arrest and induced hypothermia. Intens Care Med 2006;32:836-842.

38. Rundgren M, Westhall E, Cronberg T, Rosen I, Friberg H. Continuous amplitude-integrated electroencephalogram predicts outcome in hypothermia-treated cardiac arrest patients. Crit Care Med 2010;38:1838-1844.
39. Rossetti AO, Oddo M, Liaudet L, Kaplan PW. Predictors of awakening from postanoxic status epilepticus after therapeutic hypothermia. Neurology 2009;72:744-749.

40. Wennervirta JE, Ermes MJ, Tiainen SM, et al. Hypothermiatreated cardiac arrest patients with good neurological outcome differ early in quantitative variables of EEG suppression and epileptiform activity. Crit Care Med 2009;37:24272435.

41. Ermes M, Sarkela M, van Gils M, Wennervirta J, Vakkuri A, Salmi T. Prediction of poor outcome using detector of epileptiform EEH in ICU patients resuscitated after cardiac arrest. Conf Proc IEEE Eng Med Biol Soc 2007;2007:3056-3059.

42. Mecarelli O, Pro S, Randi F, et al. EEG patterns and epileptic seizures in acute phase stroke. Cerebrovasc Dis 2011;31:191-198.

43. Reith J, Jorgensen HS, Nakayama H, Raaschou HO, Olsen TS. Seizures in acute stroke: predictors and prognostic significance. The Copenhagen stroke study. Stroke 1997;28:1585-1589.

44. Cillessen JP, van Huffelen AC, Kappelle LJ, Algra A, van Gijn J. Electroencephalography improves the prediction of functional outcome in the acute stage of cerebral ischemia. Stroke 1994;25:1968-1972.

45. Cuspineda E, Machado C, Aubert E, Galan L, Llopis F, Avila Y. Predicting outcome in acute stroke: a comparison between qEEG and the Canadian Neurological Scale. Clin Electroencephalogr 2003;34:1-4.

46. Wood JH, Polyzoidis KS, Epstein CM, Gibby GL, Tindall GT. Quantitative EEG alterations after isovolemic-hemodilutional augmentation of cerebral perfusion in stroke patients. Neurology 1984;34:764-768.

47. Gross CE, Bednar MM, Lew SM, Florman JE, Kohut JJ. Preoperative volume expansion improves tolerance to carotid artery cross-clamping during endarterectomy. Neurosurgery 1998;43:222228.

48. Suzuki A, Yoshioka K, Yasui N. Clinical application of EEG topography in cerebral ischemia: Detection of functional reversibility and hemodynamics. Brain Topogr 1990;3:167-174.

49. Dalmau J. Status epilepticus due to paraneoplastic and nonparaneoplastic encephalitides. Epilepsia 2009;50(suppl 12):58-60.

50. Andrade DM, Tai P, Dalmau J, Wennberg R. Tonic seizures: a diagnostic clue of anti-1GI1 encephalitis? Neurology 2011;76:13551357.

51. Specchio N, Fusco L, Claps D, Vigevano F. Epileptic encephalopathy in children possibly related to immune-mediated pathogenesis. Brain Dev 2010;32:51-56.

52. Blatt I, Brenner RP. Triphasic waves in a psychiatric population: a retrospective study. J Clin Neurophysiol 1996;13:324-329.

53. Ficker DM, Westmoreland BF, Sharbrough FW. Epileptiform abnormalities in hepatic encephalopathy. J Clin Neurophysiol 1997; 14:230-234

54. Oddo M, Carrera E, Claassen J, Mayer SA, Hirsch LJ. Continuous electroencephalography in the medical intensive care unit. Crit Care Med 2009;37:2051-2056.

55. Garrett WT, Chang CW, Bleck TP. Altered mental status in thrombotic thrombocytopenic purpura is secondary to nonconvulsive status epilepticus. Ann Neurol 1996;40:245-246.

56. Kuchta J, Klug N, Ernestus RI. Nonconvulsive status epilepticus as a possible cause of coma in neurosurgical intensive care. Cen Eur Neurosurg 2009;70:176-179.

57. Cdorey LA, Pellock JM, Boggs JG, Miller LL, DeLorenzo RJ. Evidence for a genetic predisposition for status epilepticus. Neurology 1998;50:558-560.

58. Fitzpatrick W, Lowry N. Pleds: Clinical correlates. Can J Neurol Sci 2007;34:443-450.

59. Pohlmann-Eden B, Hoch DB, Cochius JI, Chiappa KH. Periodic lateralized epileptiform discharges - a critical review. J Clin Neurophysiol 1996;13:519-530. 
60. Reiher J, Rivest J, Grand'Maison F, Leduc CP. Periodic lateralized epileptiform discharges with transitional rhythmic discharges: association with seizures. Electroencephalogr Clin Neurophysiol 1991;78:12-17.

61. Kim YS, Choi SY, Kwag HJ, Kim JM. Classification and serial evolution of PLEDs. J Clin Neurol 2006;2:179-185.

62. Borges MA, Botos HJ, Bastos RF, Godoy MF, Marchi NS. Emergency EEG: study of survival. Arquivos de neuropsiquiatria 2010;68:174-178.

63. DeLorenzo RJ, Waterhouse EJ, Towne AR, et al. Persistent nonconvulsive status epilepticus after the control of convulsive status epilepticus. Epilepsia 1998;39:833-840.

64. Claassen J, Lokin JK, Fitzsimmons BF, Mendelsohn FA, Mayer SA. Predictors of functional disability and mortality after status epilepticus. Neurology 2002;58:139-142.

65. Hirsch LJ, Claassen J, Mayer SA, Emerson RG. Stimulus-induced rhythmic, periodic, or ictal discharges (SIRPIDs): a common EEG phenomenon in the critically ill. Epilepsia 2004;45:109-123.

66. Yamamoto M, Hosokawa K. Triphasic spike-wave stupor in portal-systemic encephalopathy: a case report. J Neurol Neurosurg Psychiatry 1985;48:386-387.

67. Kuroiwa Y, Celesia GG. Clinical significance of periodic EEG patterns. Arch Neurol 1980;37:15-20.

68. Neufeld MY, Korczyn AD. Topographic distribution of the periodic discharges in creutzfeldt-jakob disease (CJD). Brain Topogr 1992;4:201-206.

69. Nowack WJ, King JA. Triphasic waves and spike wave stupor. Clinical EEG (electroencephalography) 1992;23:100-104.

70. Lokrantz CM, Eriksson B, Rosen I, Asztely F. Hyperammonemic encephalopathy induced by a combination of valproate and pivmecillinam. Acta Neurol Scand 2004;109:297-301.

71. Kaplan PW, Rossetti AO. EEG patterns and imaging correlations in encephalopathy: encephalopathy part II. J Clin Neurophysiol 2011;28:233-251.

72. Aguglia U, Gambardella A, Oliveri RL, Lavano A, Quattrone A. Nonmetabolic causes of triphasic waves: a reappraisal. Clinical EEG (electroencephalography) 1990;21:120-125.

73. Martinez-Rodriguez JE, Barriga FJ, Santamaria J, et al. Nonconvulsive status epilepticus associated with cephalosporins in patients with renal failure. Am J Med 2001;111:115-119.

74. Husain AM, Mebust KA, Radtke RA. Generalized periodic epileptiform discharges: etiologies, relationship to status epilepticus, and prognosis. J Clin Neurophysiol 1999;16:51-58.

75. Dreier JP, Major S, Manning A, et al. Cortical spreading ischaemia is a novel process involved in ischaemic damage in patients with aneurysmal subarachnoid haemorrhage. Brain 2009;132:18661881

76. Shin HK, Dunn AK, Jones PB, Boas DA, Moskowitz MA, Ayata C. Vasoconstrictive neurovascular coupling during focal ischemic depolarizations. J Cereb Blood Flow Metab 2006;26:1018-1030.

77. Back T, Ginsberg MD, Dietrich WD, Watson BD. Induction of spreading depression in the ischemic hemisphere following experimental middle cerebral artery occlusion: Effect on infarct morphology. J Cereb Blood Flow Metab 1996;16:202-213.

78. Hartings JA, Strong AJ, Fabricius M, et al. Spreading depolarizations and late secondary insults after traumatic brain injury. J Neurotrauma 2009;26:1857-1866.

79. Rogatsky GG, Sonn J, Kamenir Y, Zarchin N, Mayevsky A. Relationship between intracranial pressure and cortical spreading depression following fluid percussion brain injury in rats. J Neurotrauma 2003;20:1315-1325.

80. Dreier JP, Woitzik J, Fabricius M, et al. Delayed ischaemic neurological deficits after subarachnoid haemorrhage are associated with clusters of spreading depolarizations. Brain 2006;129:32243237.
81. Bosche B, Graf R, Ernestus RI, et al. Recurrent spreading depolarizations after subarachnoid hemorrhage decreases oxygen availability in human cerebral cortex. Ann Neurol 2010;67:607617.

82. Viola S, Viola P, Litterio P, Buongarzone MP, Fiorelli L. Pathophysiology of migraine attack with prolonged aura revealed by transcranial doppler and near infrared spectroscopy. Neurol Sci 2010;31(suppl 1):S165-S166.

83. Kohl M, Lindauer U, Dirnagl U, Villringer A. Separation of changes in light scattering and chromophore concentrations during cortical spreading depression in rats. Optics Letters 1998;23:555-557.

84. Wolf T, Lindauer U, Reuter U, et al. Noninvasive near infrared spectroscopy monitoring of regional cerebral blood oxygenation changes during peri-infarct depolarizations in focal cerebral ischemia in the rat. J Cereb Blood Flow Metab 1997;17:950954.

85. Chen S, Feng Z, Li P, Jacques SL, Zeng S, Luo Q. In vivo optical reflectance imaging of spreading depression waves in rat brain with and without focal cerebral ischemia. J Biomed Opt 2006;11:34002.

86. Holtkamp M, Masuhr F, Harms L, Einhaupl KM, Meierkord H, Buchheim $\mathrm{K}$. The management of refractory generalised convulsive and complex partial status epilepticus in three European countries: a survey among epileptologists and critical care neurologists. J Neurol Neurosurg Psychiatry 2003;74:1095-1099.

87. Chong DJ, Hirsch LJ. Which EEG patterns warrant treatment in the critically ill? Reviewing the evidence for treatment of periodic epileptiform discharges and related patterns. J Clin Neurophysiol 2005;22:79-91.

88. Aranda A, Foucart G, Ducasse JL, Grolleau S, McGonigal A, Valton L. Generalized convulsive status epilepticus management in adults: a cohort study with evaluation of professional practice. Epilepsia 2010;51:2159-2167.

89. Mahmoudian T, Zadeh MM. Comparison of intranasal midazolam with intravenous diazepam for treating acute seizures in children. Epilepsy Behav 2004;5:253-255.

90. Gamez-Leyva G, Aristin JL, Fernandez E, Pascual J. Experience with intravenous levetiracetam in status epilepticus: A retrospective case series. CNS Drugs 2009;23:983-987.

91. Kellinghaus C, Berning S, Immisch I, et al. Intravenous lacosamide for treatment of status epilepticus. Acta Neurol Scand 2010;123:137-141.

92. Bensalem MK, Fakhoury TA. Topiramate and status epilepticus: report of three cases. Epilepsy Behav 2003;4:757-760.

93. Towne AR, Garnett LK, Waterhouse EJ, Morton LD, DeLorenzo RJ. The use of topiramate in refractory status epilepticus. Neurology 2003;60:332-334.

94. Rossetti AO, Milligan TA, Vulliemoz S, Michaelides C, Bertschi $\mathrm{M}$, Lee JW. A randomized trial for the treatment of refractory status epilepticus. Neurocrit Care 2010;14:4-10.

95. Krishnamurthy KB, Drislane FW. Depth of EEG suppression and outcome in barbiturate anesthetic treatment for refractory status epilepticus. Epilepsia 1999;40:759-762.

96. Claassen J, Hirsch LJ, Emerson RG, Mayer SA. Treatment of refractory status epilepticus with pentobarbital, propofol, or midazolam: A systematic review. Epilepsia 2002;43:146-153.

97. DeGiorgio CM, Heck CN, Rabinowicz AL, Gott PS, Smith T, Correale J. Serum neuron-specific enolase in the major subtypes of status epilepticus. Neurology 1999;52:746-749.

98. Correale J, Rabinowicz AL, Heck CN, Smith TD, Loskota WJ, DeGiorgio CM. Status epilepticus increases CSF levels of neuron-specific enolase and alters the blood-brain barrier. Neurology 1998;50:1388-1391.

99. DeGiorgio CM, Gott PS, Rabinowicz AL, Heck CN, Smith TD, Correale JD. Neuron-specific enolase, a marker of acute neuronal 
injury, is increased in complex partial status epilepticus. Epilepsia 1996;37:606-609.

100. Chatzikonstantinou A, Gass A, Forster A, Hennerici MG, Szabo K. Features of acute DWI abnormalities related to status epilepticus. Epilepsy Res 2011;97(1-2):45-51.

101. Di Bonaventura C, Bonini F, Fattouch J, Mari F, Petrucci S, Carni $\mathrm{M}$ et al. Diffusion-weighted magnetic resonance imaging in patients with partial status epilepticus. Epilepsia 2009;50(suppl 1):45-52.

102. Milligan TA, Zamani A, Bromfield E. Frequency and patterns of MRI abnormalities due to status epilepticus. Seizure 2009;18:104108.

103. During MJ, Fried I, Leone P, Katz A, Spencer DD. Direct measurement of extracellular lactate in the human hippocampus during spontaneous seizures. J Neurochem 1994;62:2356-2361.

104. Park S, Roederer A, Mani R, et al. Limitations of threshold-based brain oxygen monitoring for seizure detection. Neurocrit Care 2011;15(3):469-476.

105. Claassen J. How I treat patients with EEG patterns on the ictal-interictal continuum in the Neuro ICU. Neurocrit Care 2009;11:437-444.

106. Oertel M, Kelly DF, Lee JH, Glenn TC, Vespa PM, Martin NA. Metabolic suppressive therapy as a treatment for intracranial hypertension-why it works and when it fails. Acta Neurochir Suppl 2002;81:69-70.

107. Sundt TM, Jr., Sharbrough FW, Anderson RE, Michenfelder JD. Cerebral blood flow measurements and electroencephalograms during carotid endarterectomy. J Neurosurg 1974;41:310-320.

108. Labar DR, Fisch BJ, Pedley TA, Fink ME, Solomon RA. Quantitative EEG monitoring for patients with subarachnoid hemorrhage. Electroencephalogr Clin Neurophysiol 1991;78:325-332.

109. Claassen J, Hirsch LJ, Kreiter KT, et al. Quantitative continuous EEG for detecting delayed cerebral ischemia in patients with poor-grade subarachnoid hemorrhage. Clin Neurophysiol 2004;115:2699-2710.

110. Vespa PM, Nuwer MR, Juhasz C, et al. Early detection of vasospasm after acute subarachnoid hemorrhage using continuous EEG ICU monitoring. Electroencephalogr Clin Neurophysiol 1997;103:607-615.

111. Maynard D, Prior PF, Scott DF. Device for continuous monitoring of cerebral activity in resuscitated patients. Br Med J 1969;4:545546.

112. Finnigan SP, Rose SE, Walsh M, et al. Correlation of quantitative EEG in acute ischemic stroke with 30-day NIHSS score: comparison with diffusion and perfusion MRI. Stroke 2004;35:899-903.

113. Finnigan SP, Walsh M, Rose SE, Chalk JB. Quantitative EEG indices of sub-acute ischaemic stroke correlate with clinical outcomes. Clin Neurophysiol 2007;118:2525-2532.

114. Van Putten MJ, Peters JM, Mulder SM, de Haas JA, Bruijninckx CM, Tavy DL. A brain symmetry index (BSI) for online EEG monitoring in carotid endarterectomy. Clin Neurophysiol 2004;115:1189-1194.

115. Grefkes C, Fink GR. Reorganization of cerebral networks after stroke: New insights from neuroimaging with connectivity approaches. Brain 2011;134:1264-1276.
116. Yourganov G, Schmah T, Small SL, Rasmussen PM, Strother SC. Functional connectivity metrics during stroke recovery. Arch Ital Biol 2010;148:259-270.

117. Stuart RM, Waziri A, Weintraub D, et al. Intracortical EEG for the detection of vasospasm in patients with poor-grade subarachnoid hemorrhage. Neurocrit Care 2010;13:355-358.

118. Carrera E, Schmidt JM, Oddo M, et al. Transcranial doppler for predicting delayed cerebral ischemia after subarachnoid hemorrhage. Neurosurgery 2009;65:316-323.

119. Wozniak MA, Sloan MA, Rothman MI, et al. Detection of vasospasm by transcranial Doppler sonography. The challenges of the anterior and posterior cerebral arteries. J Neuroimag 1996;6:8793.

120. Proust F, Debono B, Gerardin E, et al. Angiographic cerebral vasospasm and delayed ischemic deficit on anterior part of the circle of Willis. Usefulness of transcranial Doppler. NeuroChirurgie 2002;48:489-499.

121. Houlden DA, Li C, Schwartz ML, Katic M. Median nerve somatosensory evoked potentials and the Glasgow Coma Scale as predictors of outcome in comatose patients with head injuries. Neurosurgery 1990;27:701-708.

122. Robinson LR, Micklesen PJ, Tirschwell DL, Lew HL. Predictive value of somatosensory evoked potentials for awakening from coma. Crit Care Med 2003;31:960-967.

123. Houlden DA, Taylor AB, Feinstein A, et al. Early somatosensory evoked potential grades in comatose traumatic brain injury patients predict cognitive and functional outcome. Crit Care Med 2010;38:167-174.

124. Christophis P. The prognostic value of somatosensory evoked potentials in traumatic primary and secondary brain stem lesions. Zentralbl Neurochir 2004;65:25-31.

125. Moulton RJ, Shedden PM, Tucker WS, Muller PJ. Somatosensory evoked potential monitoring following severe closed head injury. Clin Invest Med 1994;17:187-195.

126. Amantini A, Fossi S, Grippo A, et al. Continuous EEG-SSEP monitoring in severe brain injury. Clin Neurophysiol 2009;39:8593.

127. Curt A, Dietz V. Electrophysiological recordings in patients with spinal cord injury: Significance for predicting outcome. Spinal Cord 1999;37:157-165.

128. Li C, Houlden DA, Rowed DW. Somatosensory evoked potentials and neurological grades as predictors of outcome in acute spinal cord injury. J Neurosurg 1990;72:600-609.

129. Spiess M, Schubert M, Kliesch U, Halder P. Evolution of tibial SSEP after traumatic spinal cord injury: Baseline for clinical trials. Clin Neurophysiol 2008;119:1051-1061.

130. Moulton RJ, Brown JI, Konasiewicz SJ. Monitoring severe head injury: a comparison of EEG and somatosensory evoked potentials. Can J Neurol Sci 1998;25:S7-S11.

131. Oddo M, Rossetti AO. Predicting neurological outcome after cardiac arrest. Curr Opin Crit Care 17:254-259.

132. Bouwes A, Binnekade JM, Zandstra DF, et al. Somatosensory evoked potentials during mild hypothermia after cardiopulmonary resuscitation. Neurology 2009;73:1457-1461. 